\title{
Colloidal Chemistry to Advance Studies in Artificial Photosynthesis
}

\author{
Raffaella Buonsanti
}

\begin{abstract}
This article presents an overview of our research in the field of colloidal nanocrystal synthesis and their implementation into water splitting and $\mathrm{CO}_{2}$ reduction electrochemical cells. We discuss our approaches to tailormade novel material platforms to advance our knowledge in energy storage in chemical bonds, namely artificial photosynthesis. Herein, we focus on complex metal oxides as light absorbers to drive water splitting, nanocrystal hybrids and metals as electrocatalysts for carbon dioxide conversion. Our approach to solve the synthetic challenges so to achieve very precise control on size, shape and composition of such materials is highlighted.
\end{abstract}

Keywords: $\mathrm{CO}_{2}$ reduction · Colloidal synthesis · Device assembly · Nanocrystals · Water splitting

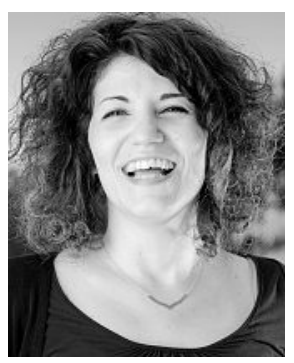

Raffaella Buonsanti has started her position as Tenure-Track Assistant Professor at the Institute of Chemical Sciences and Engineering (ISIC) of the École polytechnique fédérale de Lausanne (EPFL) in November 2015. She received her Master Degree in Chemistry from the University of Bari in 2006. In 2010, she graduated in Chemistry from the University of Salento working at the National Nanotechnology Laboratory (NNL, in Lecce-Italy). After two years as a postdoctoral researcher at LBNL (2010-2012), she was promoted to Project Scientist, a non-tenure track scientist position, in the Molecular Foundry. From 2013 to 2015 Raffaella was a tenuretrack Staff Scientist in the Department of Materials Science within the Joint Center for Artificial Photosynthesis at LBNL, before moving to EPFL. Here, through her core expertise in colloidal synthesis, she develops novel approaches to complex materials to drive chemical transformations, with particular emphasis on energy-related reactions such as $\mathrm{CO}_{2}$ reduction and water oxidation.

${ }^{\star}$ Correspondence: Prof. Dr. R. Buonsanti

EPFL Valais Wallis

EPFL SB ISCI LNCE

Rue de l'Industries 17

Case Postale 444

$\mathrm{CH}-1951$ Sion

E-mail: raffaella.buonsanti@epfl.ch

\section{Introduction}

Colloidal synthesis is a wet-chemistry approach which affords a superb control and tunability on the morphology and the composition of nanocrystalline materials (Fig 1). ${ }^{[1-10]}$ Such a material engineering at the atomic-scale is critical when searching for design specifications which will eventually lead to optimal performance in energy devices. Yet, the potential of colloidal chemistry is far from being fully explored in energy research. Nonetheless, many groups are devoting their efforts towards this direction. To cite only a few examples, Kovalenko and his team are utilizing colloidal nanocrystals to advance battery research, ${ }^{[11]}$ the Milliron group is developing a new smart window technology based on doped metal oxide nanocrystals, ${ }^{[12]}$ Wood is searching for non-toxic quantum dots for light-emitting devices, [13] Sargent continues to push the limit of quantum dot-based photovoltaics, ${ }^{[14]}$ and Cargnello is employing colloidal chemistry to design catalysts which drive high-pressure and high-temperature chemical transformations. ${ }^{[15]}$ Our group aims at exploiting the full potential of colloidal chemistry to advance energy storage in chemical bonds, otherwise named as artificial photosynthesis.

Storage of intermittent renewable energy in chemical bonds is an important research area to build a more sustainable society. Artificial photosynthesis mimics natural photosynthesis by converting sunlight, water and carbon dioxide into valueadded chemicals (such as fuels for transportation like hydrogen or hydrocarbons but also useful chemicals like ethylene, which is a feedstock for petrochemicals).

In this account, we will highlight some of the contemporary synthetic challenges in colloidal chemistry, our general approach to address them and our recent results. Firstly, we will focus on complex metal oxide nanocrystals, which are important as light absorbers to drive water oxidation among different applications. Secondly, we will discuss our very recent efforts in interfacing colloidal nanocrystals with building blocks of different chemical nature (such as metal organic frameworks) in a controlled manner. Finally, our strategies to build unambiguous structure/ properties relations in electrochemical $\mathrm{CO}_{2}$ conversion using atomically-defined nanocrystals as catalysts will be disclosed.

\section{Current Challenges in Colloidal Chemistry}

Colloidal nanocrystals (NCs) are synthesized in solution in the presence of organic surfactants (i.e. oleic acid, oleylamine, trioctylphosphine). ${ }^{[1-10]}$ The latter play a key role in colloidal synthesis. Surfactants bind unsaturated surface atoms and contribute to define the NC size and shape by modulating the surface free energy. ${ }^{[2]}$ Furthermore, they confer solubility and colloidal stability to the NCs in a large variety of solvents. Such colloidal dispersions are easily employed as 'inks' for processing of the NCs as thin film electrodes. ${ }^{[4,5,8,9,12,14]}$ At this time, the synthesis of nanocrystals containing one or two elements is pretty much mature and readily available, to the point that nanocrystals can be used as 'artificial atoms' to create nanocrystal solids. ${ }^{[16]}$ Yet, we are still far from having an extensive library of chemical reactions that permits to rationally target NCs with the desired size, shape and composition, in a similar manner of organic chemistry for example. The lack of deterministic and by-design synthetic paths becomes more significant when we go from single or binary particles to more complex 


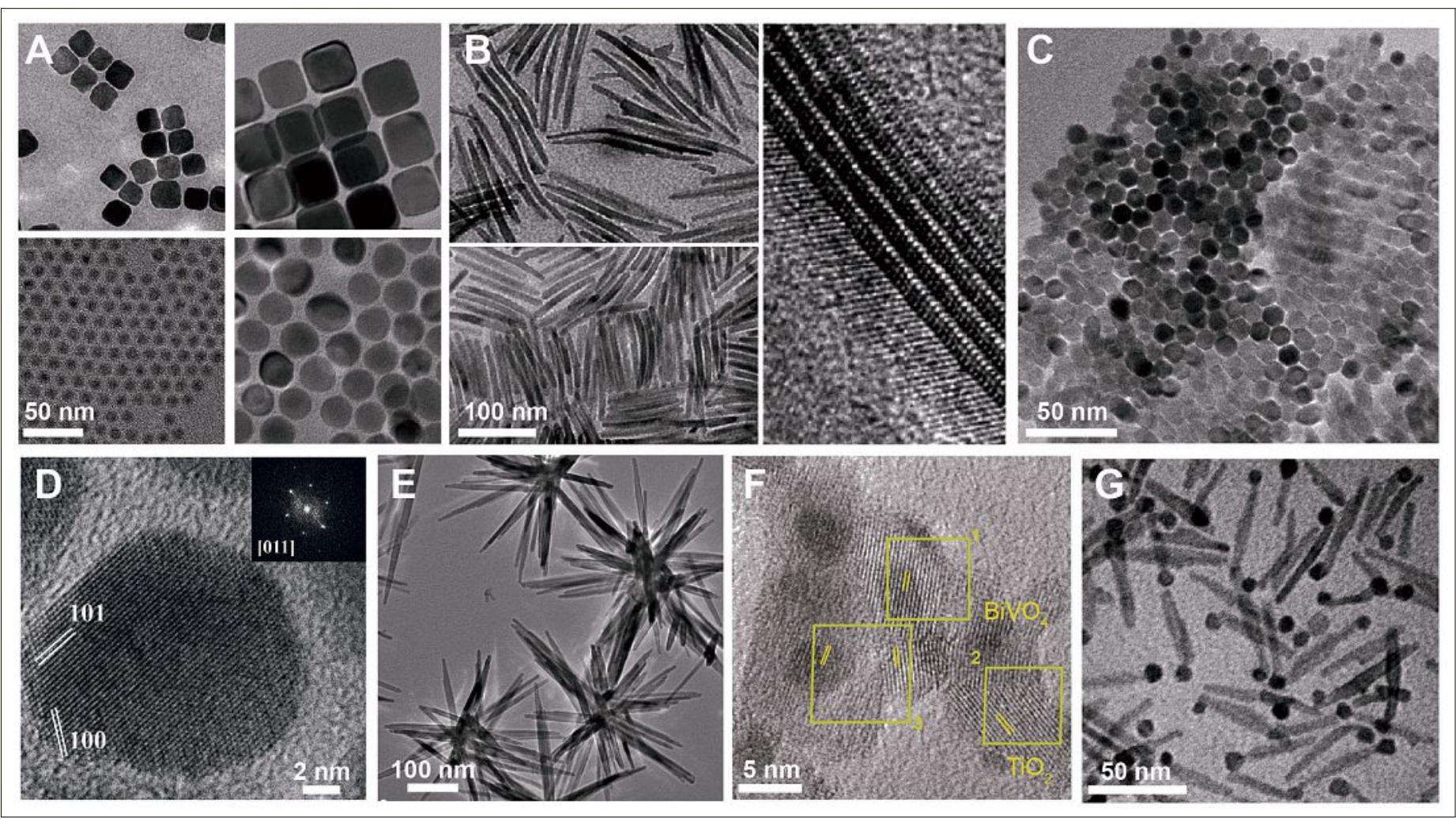

Fig. 1. Overview of transmission electron microscopy (TEM) images corresponding to colloidal nanocrystals with different composition, size and shape: (A) Cu nanospheres and nanocubes (Reprinted with permission from Angew. Chem. Int. Ed. 2016, 55, 5789. Copyright 2016 Wiley-VCH); (B) $\mathrm{TiO}_{2}$ brookite nanorods (Reprinted with permission from J. Am. Chem. Soc. 2008, 130, 11223. Copyright 2008 American Chemical Society); (C) CuZnTeSn nanorods oriented perpendicularly to the substrate; (D) Al-doped ZnO NCs (Reprinted with permission from Nano Lett. 2011, 11, 4706. Copyright 2011 American Chemical Society); (E) N-doped $\mathrm{TiO}_{2}$ nano-urchins (Reprinted with permission from J. Phys. Chem. C 2015, 119, 7443. Copyright 2015 American Chemical Society); (F) $\mathrm{BiVO}_{4} / \mathrm{TiO}_{2}$ heterostructures (Reprinted with permission from Nano. Lett. 2015, 15, 7347. Copyright 2015 American Chemical Society); (G) $\gamma$ - $\mathrm{Fe}_{2} \mathrm{O}_{3} / \mathrm{TiO}_{2} \mathrm{NC}$ hybrids (Reprinted with permission from J. Am. Chem. Soc. 2010, 132, 2437. Copyright 2015 American Chemical Society)

compositions. The latter comprise ternary, quaternary and quinary $\mathrm{NCs}$ as well as hybrid constructs. The specific synthetic challenges associated with multinary metal oxides and hybrids are described in the following sections.

Presently, our strategy to progress toward a synthesis-by-design of complex nanomaterials is to provide a better understanding of the chemical transformations behind nucleation and growth of the NCs (Fig. 2). We aim at achieving this goal through a combination of spectroscopic techniques spanning from liquid-NMR (Nuclear Magnetic Resonance) to in situ ATR-FTIR (Attenuated Total ReflectanceFourier Transform Infrared) spectroscopies. Such chemical understanding will be complemented with in situ XRD studies, which will give insight into nucleation and growth mechanism from a structural standpoint.

\subsection{Multicomponent Metal Oxide Nanocrystals}

Colloidal synthesis of metal oxide NCs is one of the core expertises of our group. We are currently relaying this knowledge to develop complex metal oxide light absorbers to drive the water oxidation reaction.

Water oxidation plays a key role in artificial photosynthesis. In fact, it is the only scalable anodic reaction to pair with the cathodic fuel-forming reactions $\left(\mathrm{H}_{2} \mathrm{O}\right.$ reduction to hydrogen fuel and $\mathrm{CO}_{2}$ reduction to value-added chemicals). Yet, light absorbers that can efficiently convert photons into charge carriers that drive this oxidative chemistry, while being stable in

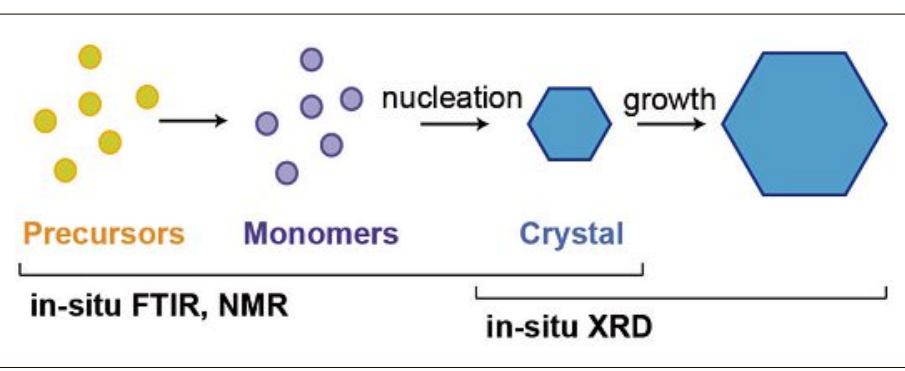

Fig. 2. Simplified schematic of nanocrystal nucleation and growth and miscellanea of techniques that our group will use to study those. the harsh environment required to carry the water oxidation reaction, are largely missing.

Many of the promising candidates suggested by theoretical calculations and some experimental results consist of multication oxides (see discussion below). The synthetic challenge associated with accessing these materials with systematic and precise definition of both composition and morphology is significant; it relies on establishing control over the reactivities of three or more metal precursors and on identifying the conditions required to form composition and structure with the desired function, without phase segregation occurring. In the context of complex multielement materials, colloidal chemistry has allowed for much progress in the class of metal chalcogenides (i.e. CuZnSSe, $\mathrm{CuZnSnS}, \mathrm{PbSeS}, \mathrm{CuZnTeSe}$ ), which have been widely used for ink-based photovoltaics. ${ }^{[17]}$ Synthetic routes to achieve an exquisite control on crystalline phases, stoichiometry, size and shape have been established and these structural properties demonstrated to correlate with the photovoltaic performance. ${ }^{[17]}$ Specifically, the superior control on phase transformations, grain size and grain orientation compared to vacuum-based methods and other molecular 
solution approaches has often resulted in much higher performance devices for this class of materials. ${ }^{[17]}$ More recently Yarema et al. have been developing and elucidating the chemistry to access ternary I-II-VI NC light emitters. ${ }^{[13]}$ On the contrary, the synthesis of ternary and quaternary oxide $\mathrm{NCs}$ is far less developed, despite the applications of this material class into a broader range of applications ranging from catalysis, to batteries and memory devices. ${ }^{[18]}$ The aim of our group is to contribute to develop the chemistry of complex oxide colloidal NCs by focusing on specific materials to advance the field of solar-tofuels conversion. If successful, our work will open the pathway to photo-physical investigation in a similar manner to the on-going studies for colloidal II-VI NCs and their hetero-structures with hydrogen evolution catalysts. ${ }^{[19]}$ In the future, dual bed baggie-reactors consisting of colloidal nanocrystals may be envisioned. ${ }^{[20]}$

The first class of interest in the group is represented by oxynitrides (i.e. $\mathrm{TiO}_{3} \mathrm{O}_{3} \mathrm{~N}_{2}$, $\mathrm{La}_{2} \mathrm{TiO}_{2} \mathrm{~N}_{2}, \mathrm{Li}_{5} \mathrm{MoO}_{4} \mathrm{~N}$ ), which have been identified as promising photoanodes.[21] They have smaller band gaps than the corresponding metal oxides because of the valence band lifting resulting from the substitution of oxygen $2 p$ orbitals with nitrogen $2 p$ levels. One of the most common approaches to oxynitrides and nitrogen doping remains high temperature annealing in ammonia. Such an approach does not allow for control on the nitrogen content, on the $\mathrm{N}$-atom location (interstitial vs. substitutional, surface vs. bulk). These parameters have all been identified to impact the electronic structure and photoactivity of the N-doped oxides and oxynitrides. ${ }^{[8]}$ Thus, achieving control on those would be desirable to establish unambiguous structure/properties correlations. We have started to explore a new chemistry for this class of light absorbers by starting from the simplest system: $\mathrm{N}$-doped $\mathrm{TiO}_{2}{ }^{[8]}$ The vast amount of literature on $\mathrm{N}-\mathrm{TiO}_{2}$ provided us a knowledge base for unambiguous verification of our synthetic approach through established spectroscopic methods. We have proposed a colloidal-based route where the rational selection of reaction conditions and precursors has allowed for the tuning of the nitrogen dopant concentration (over a wider range of concentrations compared to previous work) and, more importantly, lattice sites in $\mathrm{TiO}_{2}$. We found that ternary amines favor substitutional nitrogen and overall higher doping content compared with primary amines. A nucleation-doping mechanism, triggered by tertiary amines, and a growth-doping mechanism, which dominates when primary amines are used instead, were hypothesized to account for the introduction of nitrogen impurities in substitutional or in- terstitial sites, respectively. In the former, the Ti-N bond forms during the nucleation phase; instead in growth-doping the nitrogen dopants are mainly introduced during the growth as a result of the generation of ammonia in the reaction flask. The reaction scheme is depicted in Fig. 3 and it was supported by FTIR and NMR investigations. ${ }^{[8]}$ This newly developed chemistry allowed us to correlate the change of the band gap and the photoelectrochemical behavior of the doped $\mathrm{TiO}_{2}$ nanostructures to the nature of the dopants. The substitutionally doped $\mathrm{TiO}_{2}$ possessed a much higher activity under visible light compared to the interstitially doped sample. This result suggested that the band gap reduction in the substitutionally doped $\mathrm{TiO}_{2}$ is accompanied by actual delocalized valence band states that enable generation and transport of chemically active charges upon visible light illumination. Instead, probably only intra-band defects are introduced when nitrogen occupies interstitial lattice sites. Our aim is now to exploit the full potential of this chemistry and to demonstrate that it can be extended to a wider range of oxynitride materials.

Our second class of interest is represented by ternary and quaternary vanadates. In the last years, monoclinic $\mathrm{BiVO}_{4}$ has attracted much attention as a photoanode for solar fuel applications. ${ }^{[22]}$ The best performance for this material has been obtained by nanostructuring and by interfacing with wider band gap oxide semiconductors. ${ }^{[22]}$ We have developed a $\mathrm{BiVO}_{4} \mathrm{NC}$ ink that facilitates the assembly of $\mathrm{BiVO}_{4} /$ metal oxide $(\mathrm{MO})\left(\mathrm{MO}=\mathrm{TiO}_{2}, \mathrm{WO}_{3}\right.$, and $\left.\mathrm{Al}_{2} \mathrm{O}_{3}\right)$ nanocomposites in which the morphology of the metal oxide building blocks is finely tailored (Fig. 4). ${ }^{[9]}$ The $\mathrm{BiVO}_{4} \mathrm{NC}$ ink was constituted by $\mathrm{Bi}_{2} \mathrm{O}_{2.7} / \mathrm{VO}_{x}$ heterodimers. The latter were synthesized through an in situ seed-mediated growth where $\mathrm{Bi}$ nanosheets, generated in the reaction flask, act as nucleation seeds of a noncentrosymmetric vanadium oxide shell (Fig. 4A,B). We used bismuth chloride $\left(\mathrm{BiCl}_{3}\right)$ as a bismuth source and vanadyl acetylacetonate $\left(\mathrm{VO}(\mathrm{acac})_{2}\right)$ as a vanadium source, while oleylamine was used as surfactant and solvent. $\mathrm{BiCl}_{3}$ was reduced to elemental bismuth in the form of nanosheets by the amine. The reaction of the nanosheets with $\mathrm{VO}$ (acac), led then to the formation of the $\mathrm{Bi}_{2} \mathrm{O}_{27} / \mathrm{VO}_{\mathrm{x}}$ heterodimers. Tunable nanostructured $\mathrm{BiVO}_{4} / \mathrm{MO}$ were obtained by annealing thin films deposited from mixed solution of the $\mathrm{NC}$ heterodimers with $\mathrm{TiO}_{2}$, $\mathrm{Al}_{2} \mathrm{O}_{3}$ and $\mathrm{WO}_{3} \mathrm{NC}$ of different size and shape (Fig. 4C,D). We used a combination of transient absorption spectroscopy - spanning from picoseconds to second timescales - and photoelectrochemical measurements to reveal that the achieved structural tunability is key to understanding and directing charge separation, transport, and efficiency in these complex oxide heterostructured films (Fig. 4E,F). For example, in the $\mathrm{BiVO}_{4} / \mathrm{WO}_{3}$ nanocomposite, better photoelectrochemical performance was found for smaller $\mathrm{WO}_{3}$ nanorods (Fig. 4E,F). This behavior resulted from an improved efficiency of electron injection from $\mathrm{BiVO}_{4}$ to $\mathrm{WO}_{3}$ deriving from a more extended interfacial area between $\mathrm{BiVO}_{4}$ and $\mathrm{WO}_{3}$, compared to nanocomposites comprising bigger $\mathrm{WO}_{3}$ nanorods. These results highlight the significant potential

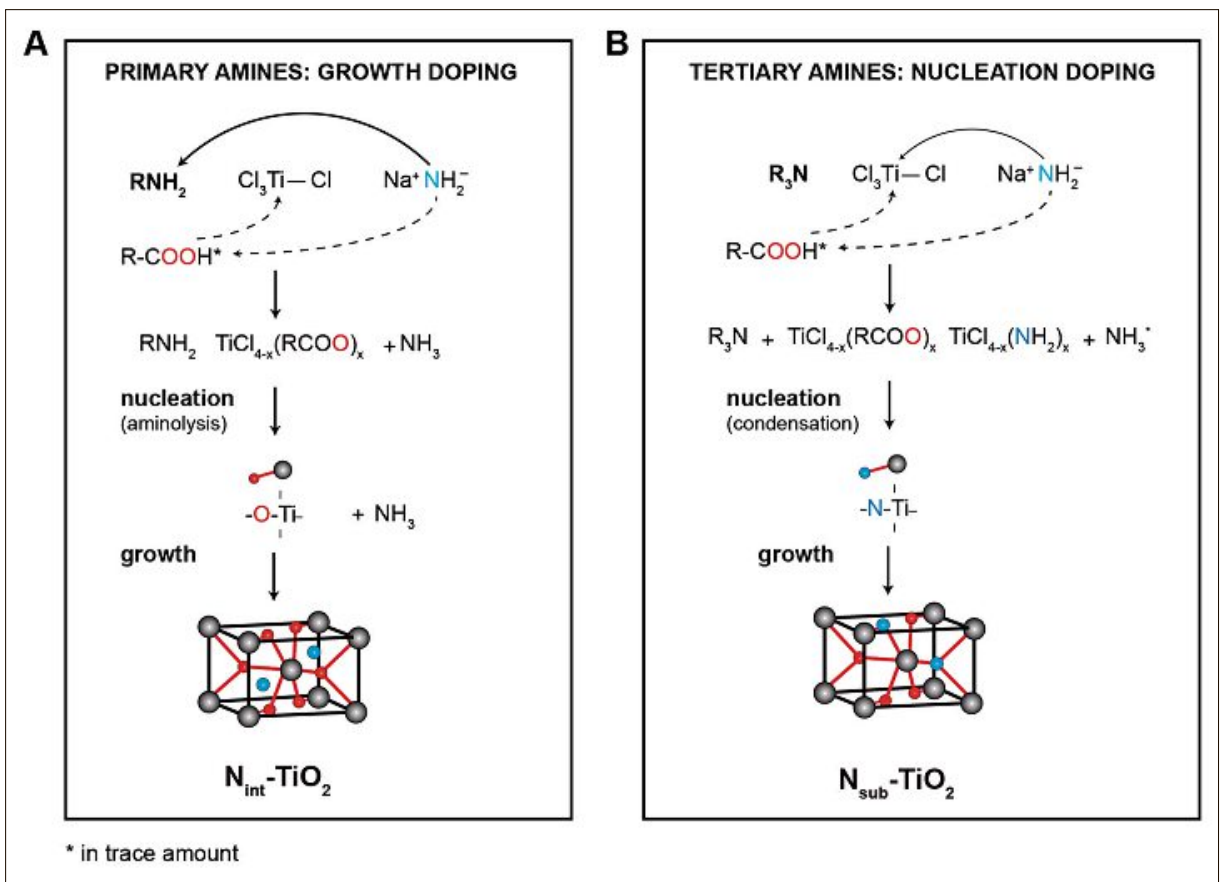

Fig. 3. Reaction schemes for the nitrogen doping of $\mathrm{TiO}_{2}$ nanostructures in A) interstitial and $\mathrm{B}$ ) substitutional lattice sites in the presence of primary and ternary amines, respectively. ${ }^{[8]}$ 
afforded by the assembly of such oxide nanocomposites and unveil relationships between MO properties and PEC performance. While the focus of this initial study was on $\mathrm{BiVO} / \mathrm{MO}$ nanocomposites, the generality of this facile method for the assembly of preformed building blocks, which avoids tedious growth optimization and long preparation times, will enable access to a wide range of tailored heterojunctions. At the same time, we learned that accessing multicomponent vanadates with uniform composition is a non-trivial task.

Balancing precursor reactivities is the key to access homogeneous composition; some understanding of the reaction mechanism is needed to properly choose the precursors. ${ }^{[23]}$ The nucleation and the growth of metal oxides are usually initiated by heating up the reaction mixture, instead of the hot-injection technique which is more common for II-VI and III-V NCs. ${ }^{[1,2]}$ A possible explanation of this different approach might rely on the reactivity of the precursors. Common precursors for metal oxides are chlorides, acetates, acetylacetonates, oleates, aminocomplexes, which often form in situ in the reaction mixture during the heating stage. Frequently used ligands include long chain (C8-C18) amines, carboxylic acids and diols. Common reaction paths for metal oxides include thermal decomposition of the precursors, aminolysis and alcolysis reactions. ${ }^{[6-8]}$ We have shown that FTIR is a great tool to corroborate hypotheses about the reaction mechanism. ${ }^{[6-8]}$

As for $\mathrm{BiVO}_{4}$, bismuth is a border line Lewis acid and vanadium is a hard Lewis acid. Therefore, bismuth acetate is expected to be less reactive than vanadium acetate. In our attempt to obtain colloidal $\mathrm{BiVO}_{4} \mathrm{NCs}$, when bismuth chloride was substituted with bismuth acetate or ethylhexanoate, $300 \mathrm{~nm}$ bismuth aggregates formed before bismuth could react with the vanadium precursor. This suggests that our hypothesis regarding reactivity was wrong and that we need to develop a deeper understanding of the chemistry behind the nucleation and growth of these vanadates. We plan to do so by looking also at different cations (such as copper or manganese), so to be able to further manipulate the band gap while elucidating the reaction mechanisms.

As an alternative approach to colloidal chemistry, we are investigating a NC-seed mediated growth of thin film vanadate light absorbers. The seeding process has been traditionally employed to reduce the crystallization temperature in ceramic ferroelectric materials, such a lead zirconate titanate, to facilitate their integration in flexible electronics as a result of the decreased crystallization temperature.[24]
Recently, our group has utilized colloidal NCs with variable composition as seeds for the nucleation and growth of vanadate thin films (Fig. 5). ${ }^{[25]}$ Specifically, we employed $\mathrm{Bi} \mathrm{Sb}_{1-\mathrm{x}}$ colloidal NCs with variable composition $x$ to discover a new light absorber, $\mathrm{BiV}_{1-\mathrm{x}} \mathrm{Sb}_{\mathrm{x}} \mathrm{O}_{4}$. The $\mathrm{Bi}_{1-\mathrm{x}} \mathrm{Sb}_{\mathrm{x}} \mathrm{NCs}$ were converted into $\mathrm{Sb}^{-\mathrm{BiVO}_{4}}$ films by reacting them with vanadyl acetyacetonate upon annealing (Fig. 5A). This novel two-step synthetic approach allowed for a precise compositional tuning while preserving the same morphology and crystalline structure up to a $20 \%$ antimony content. It is important to note that attempts to synthesize $\mathrm{Sb}-\mathrm{BiVO}_{4}$ by more conventional approaches such as sol-gel, solid solution, and spray pyrolysis were unsuccessful and resulted in phase separation, even for low percentage $\mathrm{Sb}$ alloys. X-ray characterization techniques revealed the substitution of $\mathrm{V}^{5+}$ with $\mathrm{Sb}^{5+}$ in the monoclinic $\mathrm{BiVO}_{4}$ lattice. Thanks to the broad compositional range covered by our approach, a combination of theoretical predication and experimental validation has showed that this photoanode possesses a band gap that linearly decreases with increasing Sb content up to $20 \%$ when the band gap is $0.2 \mathrm{eV}$ smaller than the one of the undoped counterpart (Fig. 5A). While this material is still far from being optimal, the results of this work, derived from close coupling of theoretical

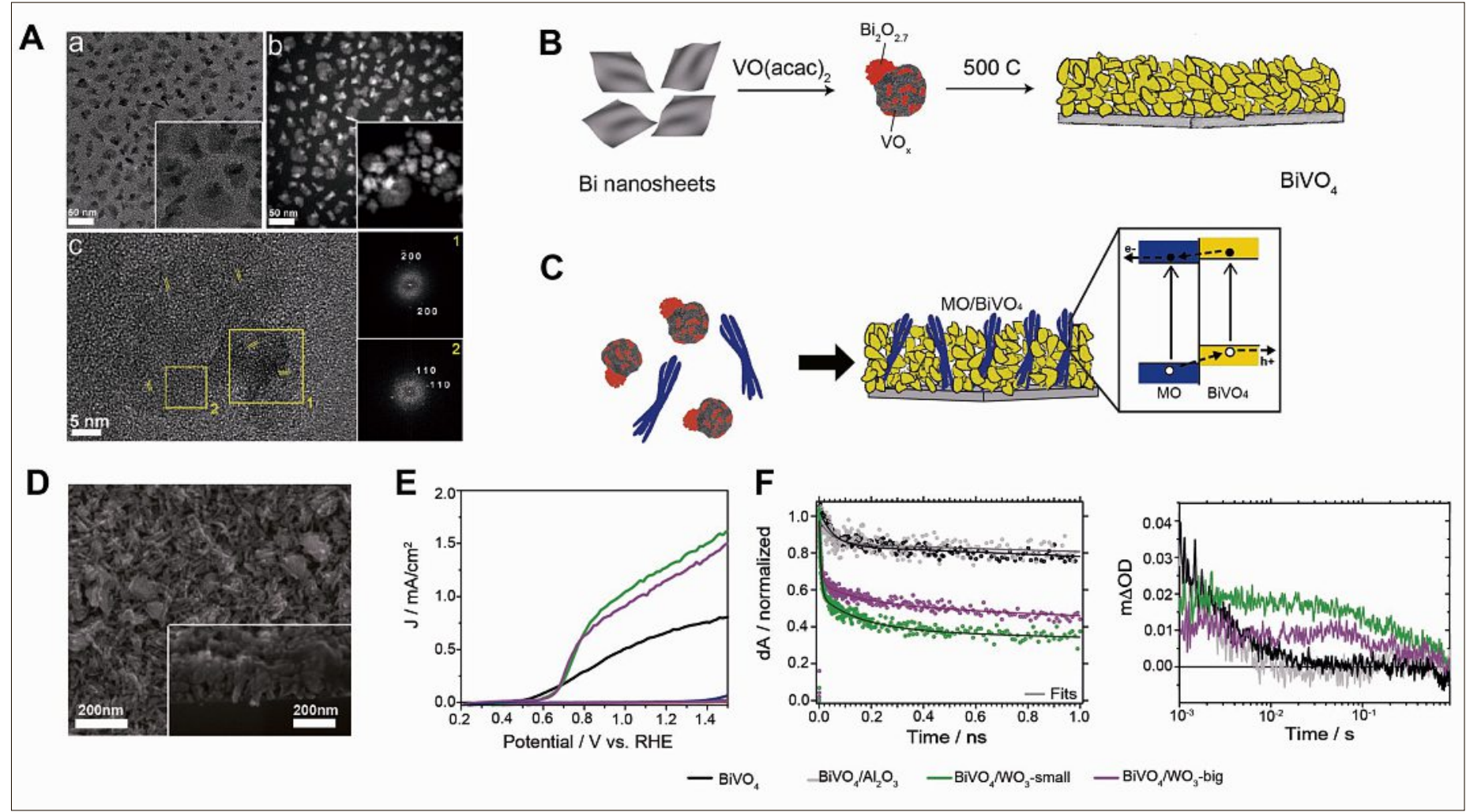

Fig. 4. Overview of assembly and photocarrier dynamics of heterostructured $\mathrm{BiVO}_{4} / \mathrm{MO}$ heterostructured nanocomposite photoanodes: $(\mathrm{A}) \mathrm{TEM}$ analysis of the heterodimers; $(\mathrm{B}, \mathrm{C})$ schematic representation of the $\mathrm{Bi}_{2} \mathrm{O}_{2.7} / \mathrm{NO}_{x}$ heterodimers and their assembly with metal oxide $\mathrm{NCs}$ to form the nanocomposites, respectively; (D) scanning electron microscopy images of $\mathrm{BiVO}_{4} / \mathrm{TiO}_{2}$ nanocomposites; (E, F) photoelectrochemical and photophysical characterization of $\mathrm{BiVO}_{4} / \mathrm{Al}_{2} \mathrm{O}_{3}$ and $\mathrm{BiVO}_{4} / \mathrm{WO}_{3}$, the latter assembled from the $\mathrm{Bi}_{2} \mathrm{O}_{2.7} / \mathrm{NO}_{x}$ heterodimers and two different sizes of WO nanorods. ${ }^{[9]}$ Reprinted with permission from Nano. Lett. 2015, 15, 7347. Copyright 2015 American Chemical Society. 
understanding and advanced synthesis approaches, provide a path to the design of other metal oxides alloys with desirable band gaps and band edge positions for solar water splitting. To demonstrate the general applicability of the NC-seeded growth approach, other high surface area ternary oxide light absorbers were targeted (Fig. 5B). $\mathrm{Bi}_{2} \mathrm{WO}_{6}, \mathrm{CuWO}_{4}, \mathrm{SnWO}_{6}$, and $\mathrm{InVO}_{4}$ were synthetized and structurally characterized by XRD by using $\mathrm{Bi}, \mathrm{Cu}, \mathrm{Sn}$ and $\mathrm{In}_{2} \mathrm{O}_{3} \mathrm{NC}$ seeds, respectively (Fig. 5B). Presently, we are targeting various quaternary vanadates, which we expect to possess a tunable band gap in the visible range. At the same time we are developing in situ techniques to gain a better understanding on the conversion mechanism of the NC seeds into the thin films. We aim at controlling grain size and orientation of the films, which are expected to impact performance, by tuning NC size and shape.

\subsection{Nanocrystal Hybrids}

The assembly of colloidal NCs with building blocks of different chemical nature (i.e. polymers, glasses and carbonbased materials) show considerable promise to satisfy the demand for complexity of several applications ranging from gas storage to energy storage to catalysis.[26,27] The functionalities of such hybrid materials are dictated not only by the intrinsic properties of the single units but also by the morphology of the hybrids, the reciprocal spatial distribution of their constituents and the interfaces between them. ${ }^{[26,27]}$ Therefore, it is crucial to develop synthetic approaches to control these features.

Our group is studying NC-based hybrids which will eventually be utilized as multifunctional catalytic platforms to advance $\mathrm{CO}_{2}$ conversion studies. The goal here is to establish novel synthetic schemes to achieve an exquisite control and tunability of these hybrid materials across multiple length. Firstly, we need to establish rules of design to synthesize such hybrids in a programmable manner.

In our recent work, we have focused on $\mathrm{NC} /$ metal organic framework (MOF) hybrids. Beautiful work has been done by the team led by Fischer based on wet- and gasimpregnation of pre-synthesized MOF. ${ }^{[28]}$ Here, small and naked NCs are embedded into the frameworks; however the control of size, shape, and composition of the NCs themselves is highly constrained by the pre-formed frameworks. Solvo-chemical conversion of metal oxide NCs developed in Falcaro's group has enabled the assembly of two- and three-dimensional MOF architectures on various substrates. ${ }^{[29]}$ Despite the demonstrated potential of chemical conversion, the synthesis of metal NC@MOFs by such an approach still

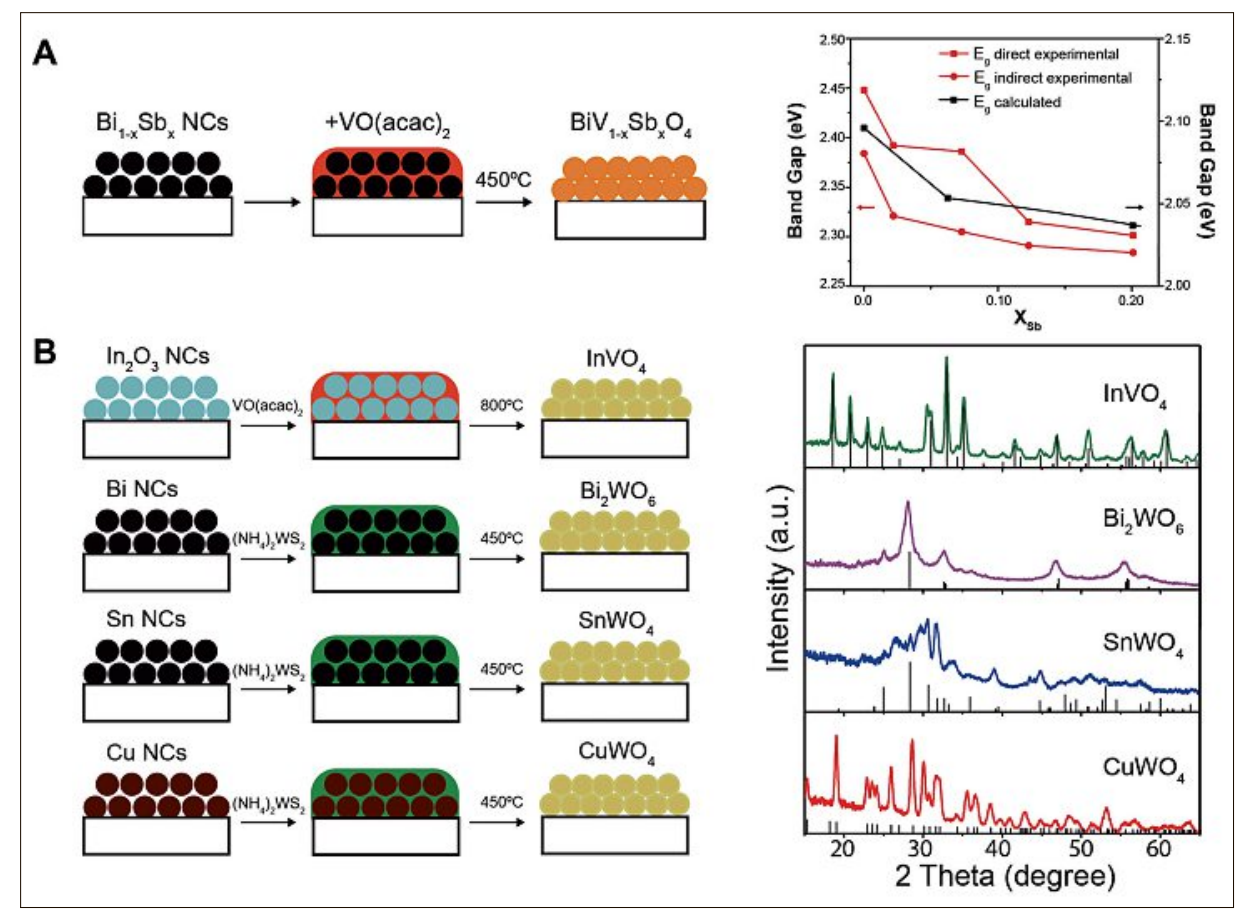

Fig. 5. NC-seed mediated growth of thin films metal oxide light absorbers: (A) schematic for growth of $\mathrm{Sb}$-alloyed $\mathrm{BiVO}_{4}$ and corresponding theoretical and experimental band gap variation; (B) applicability of the NC-seeded growth to other metal oxides. ${ }^{[25]}$ Reprinted with permission from Adv. Mater. 2015, 27, 6733. Copyright 2015 Wiley-VCH.

remains an underexplored area compared to the oxide counterpart.

We have reported a one-step synthetic strategy to encapsulate ligand-free (naked) $\mathrm{Cu}$ NCs into a carboxylate based $\mathrm{MOF}(\mathrm{Cu}-$ MOF-74).[30] Cu@Cu-MOF-74 hybrids were synthesized via a partial conversion of naked $\mathrm{Cu} \mathrm{NCs}$ into $\mathrm{Cu}-\mathrm{MOF}-74$ in the presence of $\mathrm{H}_{4}$ dobdc (2,5-dihydroxyterephthalic acid, the MOF ligand) in a solvent mixture of dimethylformamide (DMF) and ethanol (EtOH) (Fig. 6).

One of the hurdles to realizing metal NC@MOF hybrids with uniform morphology and with a homogeneous NC spatial distribution by chemical transformation is to precisely match the metal dissolution and the MOF crystallization rate. Our study defined and rationalized the parameter space that provides control of the $\mathrm{NC}$ dissolution rate so to find the optimal window (Fig. 6). The initial $\mathrm{Cu}$ NCs uniformly dissolve during the conversion and this results in a narrow size distribution of the NCs in the final material. By explaining the role played by the solvent polarity, the reaction temperature, and the reactant concentration, we have contributed to gain knowledge toward a more rational approach to the synthesis of metal NC@ MOF hybrids rather than more traditional trial and error methods.

To disclose the potentiality of the $\mathrm{Cu} @ \mathrm{Cu}-\mathrm{MOF}-74$ hybrids for device integration, we have demonstrated that the seeding approach reported here works on different substrates commonly used in electrocatalysis, such as glassy carbon and carbon paper.[30] Charge transport studies and structural stability will be object of future studies.

Nowadays, a huge compositional library of NCs is accessible by colloidal chemistry and we foresee the extension of the metal NC-to-MOF conversion to NCs with different sizes and shapes and to more complex systems, such as bimetallic NCs. Furthermore, the access to a pristine interface between inorganic NCs and MOFs will pave the way towards exploring new synergistic effects at the interface, especially in catalysis or gas adsorption where surface interactions dictate the material properties.

\section{Colloidal Chemistry to Advance $\mathrm{CO}_{2}$ Conversion Studies}

$\mathrm{CO}_{2}$ is an under-utilized resource for chemicals and fuels. With gigatons of this gas released in the atmosphere each year, any technology which uses $\mathrm{CO}_{2}$ as a raw material can make a huge impact on climate change. However, the thermodynamic stability of the $\mathrm{CO}_{2}$ molecule implies that high temperatures, highly reactive reagents (i.e. hydrogen) or a different source of energy are required to carry out its conversion. The exploitation of intermittent renewable sources (i.e. solar) emerges as a win-win solution, considering the need for seasonal storage. While progress has been made, one of the main challenges is to find 


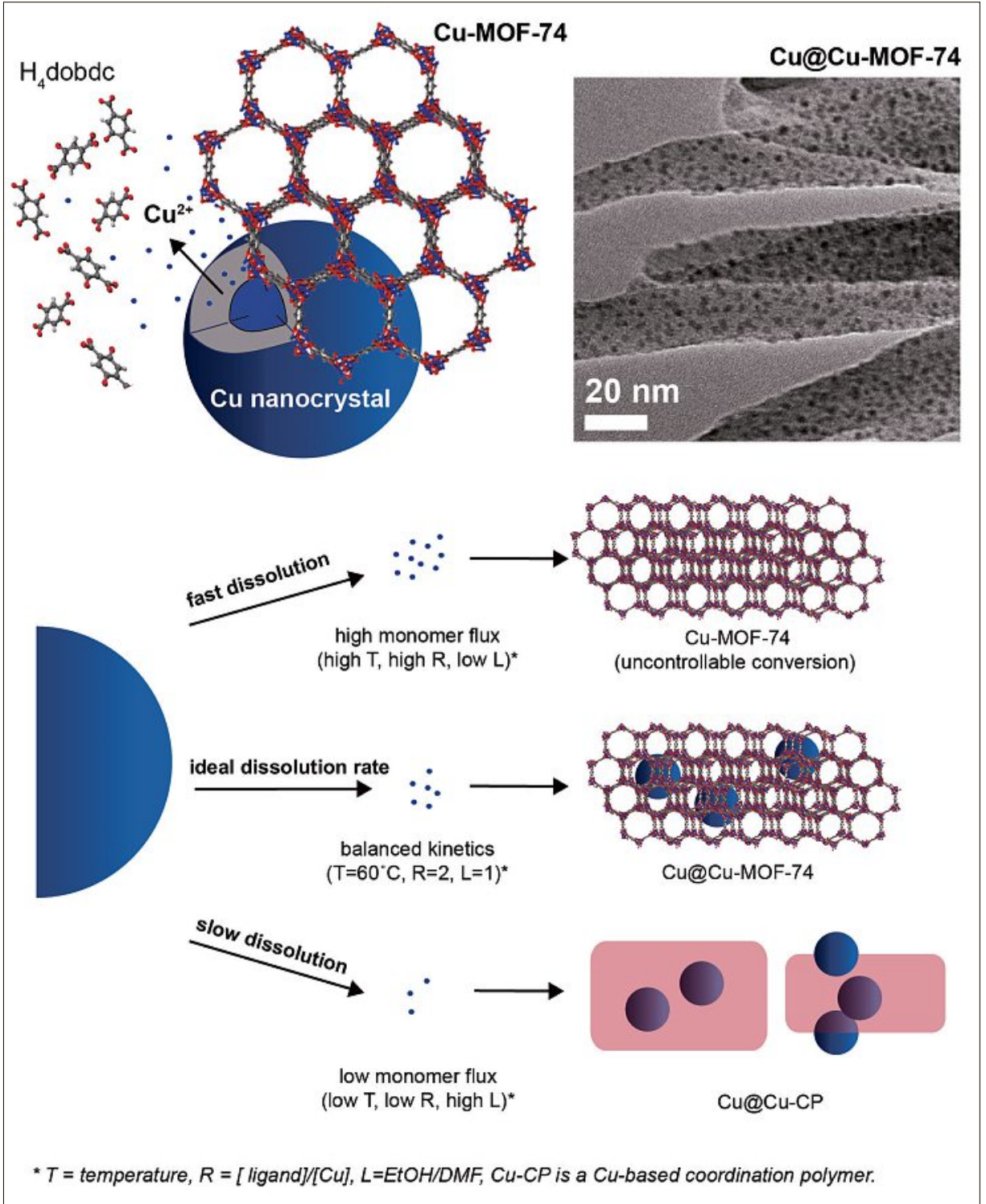

Fig. 6. Proposed formation mechanism and one representative TEM image of the Cu@CuMOF-74 hybrids (top) and impact of NC dissolution rate on the growth of the same (bottom). ${ }^{[30]}$ Reprinted with permission from Chem. Mater. 2016, 28, 3839. Copyright 2016 American Chemical Society. tion would decrease and the selectivity towards hydrocarbons would increase if these oxygen-containing intermediates are stabilized. On metal surfaces the binding energy of $\mathrm{CO}$ and the downstream oxygen containing intermediates are linearly correlated (Fig. 7C), as they all bind through the carbon atom. ${ }^{[32-34]}$ As a consequence, on pure metal surfaces, it is not possible to stabilize $\mathrm{CHO}$ without stabilizing $\mathrm{CO}$ and thus poisoning the catalyst surface. Copper is more efficient and selective than other metals only because it has a slightly better than average ability to perform the hydrogenation of the adsorbed CO. Norskov et al. have suggested that more efficient and selective catalysts should decouple the adsorption energies of $\mathrm{CO}$ and $\mathrm{CHO} \cdot{ }^{[32-34]}$

In our recent work, we have explored the effect of both size and shape of $\mathrm{Cu} \mathrm{NCs}$ on their electroreduction activity and product selectivity. ${ }^{[5]}$ It is known that catalyst morphology can modulate catalytic activity and selectivity, yet this remains a relatively underexplored area in electrochemical $\mathrm{CO}_{2}$ reduction. Two different sizes of $\mathrm{Cu} \mathrm{NC}^{2}$ spheres $(7.5 \mathrm{~nm}$ and $27 \mathrm{~nm})$ and three different sizes of $\mathrm{Cu}$ NC cubes $(24 \mathrm{~nm}$, $44 \mathrm{~nm}$, and $63 \mathrm{~nm}$ ) were synthesized by the same colloidal chemistry-based method (Fig. 7A). Within the same morphology, we found that smaller NCs exhibit higher activity; however overall, the cube-shaped NCs were more intrinsically active than the spheres. An unexpected non-monotonic selectivity trend was observed, with an $80 \%$ selectivity for carbon products, $40 \%$ of which corresponded to ethylene, for the best case $\mathrm{Cu}$ NC cubes with $44 \mathrm{~nm}$ side length (Fig. 7B). While increased selectivity for ethylene at (100) step sites of $\mathrm{Cu}$ was anticipated by previous literature, the size-dependence was quite surprising, as smaller particles are commonly the most catalysts that can electrochemically reduce $\mathrm{CO}_{2}$ with high efficiency and selectivity toward a specific product. ${ }^{[31]}$

We believe that colloidal chemistry offers a huge potential in advancing $\mathrm{CO}_{2}$ conversion studies. The material tunability afforded by such technique is ideal to build unambiguous relations between the catalyst structure and its activity and to understand the relevant parameters to modulate activity and selectivity.

Currently, $\mathrm{Cu}$ is the only catalyst with propensity to form hydrocarbons, which are high energy dense molecules and therefore desirable products. ${ }^{[31]}$ In the conversion pathway from $\mathrm{CO}_{2}$ to hydrocarbons, oxygen-containing intermediates, such as $\mathrm{CHO}$, and the hydrogenation reaction to form those, have been identified as potential limiting steps. ${ }^{[32-34]}$ This means that the reaction barrier of $\mathrm{CO}_{2}$ reduc-
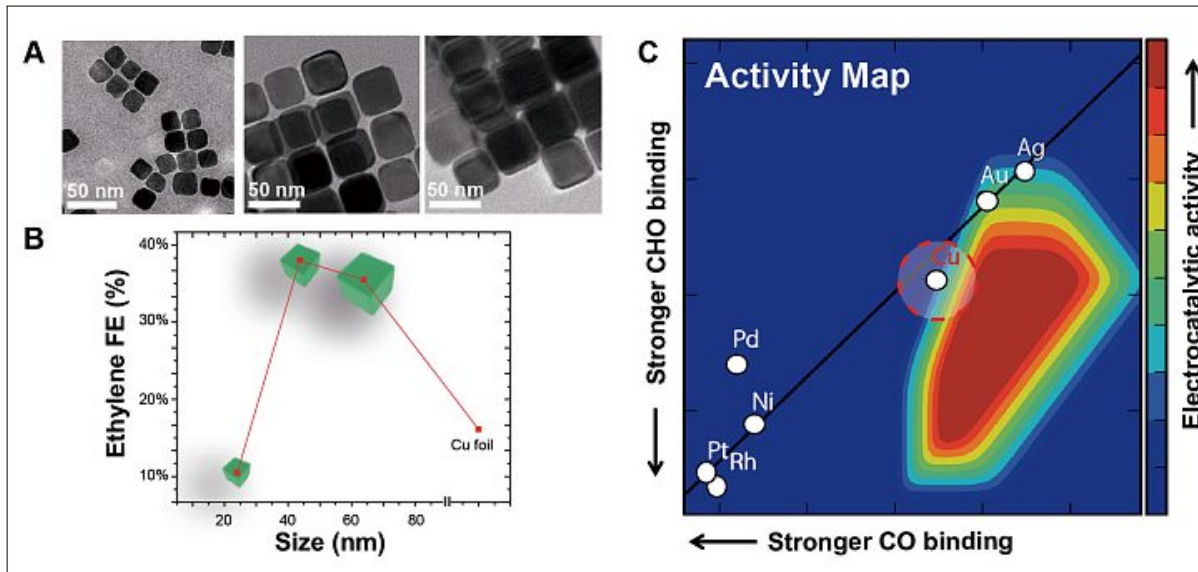

Fig. 7. Colloidal $\mathrm{Cu}$ nanocrystals as electrocatalysts for $\mathrm{CO}_{2}$ reduction: (A) TEM images of $\mathrm{Cu}$ cubes with an average edge length of 24, 44, $63 \mathrm{~nm}$; (B) corresponding faradaic efficiencies towards ethylene (the complete product analysis can be found in reference [5]); (C) kinetic volcano adapted from reference [34]. Reprinted with permission from J. Phys. Chem. Lett. 2013, 4, 388. Copyright 2013 American Chemical Society. 
reactive ones. Our hypothesis is that atoms at the edges and on the (100) planes behave as different catalytic sites and that an optimal ratio between them $\left(\mathrm{N}_{\text {edge }} / \mathrm{N}_{100}\right.$ $=0.25$ for $\mathrm{d}=44 \mathrm{~nm}$, where $\mathrm{N}$ indicates the number of atoms at the edge or on the planes) is crucial to maximize $\mathrm{CO}_{2}$ reduction products and ethylene specifically. We are now verifying such a hypothesis and also studying the morphological evolution of the catalyst over time under operational conditions.

The activity map in Fig. 7C has been calculated for ideal flat metallic surfaces. Our work and others now show that morphology allows to gain some degree of freedom on the map. Yet, we are still far from being able to tune single metal catalysts so to maximize their electrocatalytic activity towards a specific product.

In the future, the group will continue to look at colloidal catalysts with different composition and morphologies in order to gain more insights into the $\mathrm{CO}_{2}$ conversion mechanism and to maximize the hydrocarbon production. The next challenge will be to synthesize and to investigate hybrid materials with thoroughly bi-functional sites.

\section{Conclusion and Outlook}

Our research is highly multidisciplinary as it spans from synthesis of new atomically-defined materials to their implementation into proof-of-concept devices so to derive unambiguous structure/properties relations. While addressing synthetic challenges in colloidal chemistry, we focus on materials for storing energy in chemical bonds. Because we are integrating nanocrystals into electrochemical devices, we face challenges which are common in many applications involving NCs, from batteries to solar cells and smart windows. The primary one is to assure electrical connection between the NCs and the substrate and between the individual NCs. In fact, the pristine ligands are insulating. Currently, we are adopting previously developed strategies such as thermal decomposition or chemical stripping of the native ligands. One requirement, which is specific to material implementation into water splitting and $\mathrm{CO}_{2}$ electrochemical cells, is to assure their stability in water and under highly oxidative or reducing conditions. We have recently started some work on capping NC semiconductor thin films with thin metal oxide layers by atomic layer deposition as a way to passivate the surface of watersensitive materials and to explore different mechanisms of energy transport in NC networks. Overall, in the next years we aim at contributing to the broader nanoscience field with our research on colloidal NCs for energy storage into chemical bonds.

\section{Acknowledgements}

Part of the work outlined above was supported by the Joint Center for Artificial Photosynthesis, a U.S. DOE Energy Hub (Award Number DE-SC0004993), by Laboratory Directed Research and Development (LDRD) funding from Berkeley Lab (Contract No. DEAC02-05CH11231) and by the start-up funding from our host institution (EPFL). We thank the Swiss SNF (AP Energy Grant, project number PYAPP2_166897/1), the European Union (FP7 ERC Starting Grant 'HY-CAT' - just awarded), our industrial partner Gaznat and the Swiss National Center of Research (NCCR) Marvel which will all support our research in the upcoming years. Finally, I would like to acknowledge my former and present mentors, all the great colleagues I have met in Berkeley and now here in Switzerland and the wonderful group of dedicated students and postdocs I have the pleasure to work with.

Received: August 24, 2016

[1] S. G. Kwon, T. Hyeon, Acc. Chem. Res. 2008, $41,1696$.

[2] Y.-w. Jun, J.-s. Choi, J. Cheon, Angew. Chemie. Int. Ed. 2006, 45, 3414.

[3] M. Casavola, R. Buonsanti, G. Caputo, P. D Cozzoli, Eur. J. Inorg. Chem. 2008, 6, 837.

[4] M. V. Kovalenko, L. Manna, A. Cabot, Z. Hens, D. V Talapin, C. R. Kagan, V. I. Klimov, A. L. Rogach, P. Reiss, D. J. Milliron, P. GuyotSionnest, G. Konstantatos, W. J. Parak, T. Hyeon, B. A. Korgel, C. B. Murray, W. Heiss, ACS Nano 2015, 9, 1012.

[5] A. Loiudice, P. Lobaccaro, E. A. Kamali, T. Thao, B. H. Hung, J. W. Ager, R. Buonsanti, Angew. Chem. Int. Ed. 2016, 55, 5789.

[6] R. Buonsanti, V. Grillo, E. Carlino, C. Giannini, T. Kipp, R. Cingolani, P. D. Cozzoli, J. Am. Chem. Soc. 2008, 130, 11223.

[7] R. Buonsanti, A. Llordes, S. Aloni, B. Helms, D. J. Milliron, Nano Lett. 2011, 11, 4706.

[8] J. Lynch, C. Giannini, J. K. Cooper, A. Loiudice, I. D. Sharp, R. Buonsanti, J. Phys. Chem. C 2015, 119, 7443 .

[9] A. Loiudice, J. K. Cooper, L. H. Hess, T. M. Mattox, I. D. Sharp, R. Buonsanti, Nano. Lett. 2015, 15, 7347.

[10] R. Buonsanti, E. Carlino, V. Grillo, C. Giannini, F. Gozzo, M.Garcia-Hernandez, M. A. Garcia, R. Cingolani, P. D. Cozzoli, J. Am. Chem. Soc. 2010, 132, 2437.

[11] M. F. Oszajca, M. I. Bodnarchuk, M. V. Kovalenko, Chem. Mater. 2014, 26, 5422.

[12] A. Llordes, G. Garcia, J. Gazquez, D. J. Milliron, Nature 2013, 500, 323.

[13] O. Yarema, M. Yarema, D. Bozyigit, W. M. M. Lin, V. Wood, ACS Nano 2015, 9, 1134.

[14] Z. Ning, O. Voznyy, J. Pan, S. Hoogland, V. Adinolfi, J. Xu, M. Li, A. R. Kirmani, J.-P. Sun, J. Minor, K. W. Kemp, H. Dong, L. Rollny, A. Labelle, G. Carey, B. Sutherland, I. Hill, A Amassian, H. Liu, J. Tang, O. M. Bakr, E. H. Sargent, Nature Mater. 2014, 13, 822.

[15] M. Cargnello, V. Doan-Nguyen, T. R. Gordon, T. Paik, R. E. Diaz, E. A. Stach, R. J. Gorte, P. Fornasiero, C. B. Murray, Science 2013, 341, 771.

[16] M. V. Kovalenko, Chimia 2013, 67, 316.

[17] a) H. Zhou, W.-C. Hsu, H.-S. Duan, B. Bob, W. Yang, T.-B. Song, C.-J. Hsu; Y. Yang Energy Environ. Sci. 2013, 6, 2822; b) R. Mainz, A. Singh, S. Levcenko, M. Klaus, C. Genzel, K. M. Ryan, T. Unold, Nature Commun. 2014, 5 , 4133.
[18] a) M. Niederberger, G. Garnweitner, N. Pinna, M. Antonietti, J. Am. Chem. Soc. 2004, 126, 9120; b) M. D. Rossell, Q. M. Ramasse, S. D. Findlay, F. Rechberger, R. Erni, M. Niederberger, ACS Nano 2012, 6, 7077.

[19] a) T. Simon, N. Bouchonville, M. J. Berr, A. Vaneski, A. Adrović, D. Volbers, R. Wyrwich, M. Döblinger, A. S. Susha, A. L. Rogach, F. Jäckel, J. K. Stolarczyk, J. Feldmann, Nature Mater. 2014, 13, 1013; b) J. K. Utterback, A. N. Grennell, M. B. Wilker, O. M. Pearce, J. D. Eaves, G. Dukovic, Nature Chem. 2016, doi: 10.1038/nchem. 2566

[20] B. A. Pinaud, J. D. Benck, L. C. Seitz, A. J. Forman, Z. Chen, T. G. Deutsch, B. D. James, K. N. Baum, G. N. Baum, S. Ardo, H. Wang, E. Miller, T. F. Jaramillo, Energy Environ. Sci. 2013, 6, 1983.

[21] Y. Wu, P. Lazic, G. Hautier, K. Persson, G. Ceder, Energy Environ. Sci. 2013, 6, 157.

[22] a) F. F.Abdi, L. Han, A. H. M. Smets, M. Zeman, B. Dam, R. van de Krol, Nature Commun. 2013, 4, 2195; b) T. W. Kim, K.-S. Choi, Science 2014, 990, 343; c) X. Shi, I. Y. Choi, K. Zhang, J. Kwon, D. Y. Kim, J. K. Lee, S. H. Oh, J. K. Kim, J. H. Park, Nature Commun. 2014, 5 , 4775.

[23] R. Buonsanti, D. J. Milliron Chem. Mater. 2013, 25,1305 .

[24] a) I. Bretos , R. Jiménez , A. Wu , A. I. Kingon, P. M. Vilarinho, M. L. Calzada, Adv. Mater 2014, 26, 1405; b) I. Bretos, R. Jiménez, D. Pérez-Mezcua, N. Salazar, J. Ricote, M. L. Calzada, Adv. Mater. 2015, 27, 2608.

[25] A. Loiudice, J. Ma, W. S. Drisdell, T. M. Mattox, J. K. Cooper, T. Thao, C. Giannini, J. Yano, L.-W. Wang, I. D. Sharp, R. Buonsanti, Adv. Mater. 2015, 27, 6733 .

[26] a) A. Llordés, G. Garcia, J. Gazquez, D. J. Milliron, Nature 2013, 500, 323; b) K.-J. Jeon, H. R. Moon, A. M. Ruminski, B. Jiang,; C. Kisielowski, R. Bardhan, J. J. Urban, Nature Mater. 2011, 10, 286; c) M.-H. Park, C. Subramani, S. Rana, V. M. Rotello, Adv. Mater. 2012, 24, 5862 .

[27] a) G. Li, H. Kobayashi, J. M. Taylor, R. Ikeda, Y. Kubota, K. Kato, M. Takata, T. Yamamoto, S. Toh, S. Matsumura, H. Kitagawa, Nat. Mater. 2014, 13, 802; b) K. Na, K. M. Choi, O. M. Yaghi, G. A. Samorjai, Nano Lett. 2014, 14, 5979.

[28] C. Rösler, R. A. Fischer, Cryst. Eng. Comm. 2015, $17,199$.

[29] C. M. Doherty, D. Buso, A. J. Hill, S. Furukawa, S. Kitagawa, P. Falcaro, Acc. Chem. Res. 2013, 47, 396.

[30] I. Luz, A. Loiudice, D. T. Sun., W. L. Queen, R. Buonsanti, Chem. Mater. 2016, 28, 3839.

[31] a) Y. Hori, K. Kukuchi, S. Suzuki, Chem. Lett. 1985, 1695; b) C. Costentin, M. Robert, J. M. Saveant, Chem. Soc. Rev. 2013, 42, 2423; c) Y. Hori, 'Electrochemical $\mathrm{CO}_{2}$ Reduction on Metal Electrodes', in 'Modern Aspects of Electrochemistry', No 42, Eds. C. G. Vayenas, R. E. White, M. E. Gamboa Aldeco, Springer: New York, 2008, 89; d) K. P. Kuhl, E. R. Cave, D. N. Abram, T. F. Jaramillo, Energy Environ. Sci. 2012, 5, 7050; e) X. Lim, Nature 2015, 526, 628.

[32] A.A. Peterson, F. Abild-Petersen, F. Studt, J. Rossmeisl, J. K. Norskov, Energy Environ Sci. 2010, 3, 1311.

[33] A. A. Peterson, J. K. Norskov, J. Phys. Chem. Lett. 2012, 3, 251.

[34] H. A. Hansen, J. B. Varley, A. A. Peterson, J. K. Norskov, J. Phys. Chem. Lett. 2013, 4, 388. 